\title{
An Optical Method for Measuring the Diameter of Polyester Filament in Real Time
}

\author{
Masahiro UEDA, Kazuhiko ISHIKAWA,* Katsuhiko ASADA,* Tatsuyuki OTANI, \\ and Hirotsuna TANIZAWA** \\ Department of Human Ecology and Technology, Faculty of Education and Regional Studies, Fukui University \\ 3-Y-1 Bunkyo, Fukui 910-0017 \\ *Department of Information Science, Faculty of Engineering, Fukui University, 3-9-1 Bunkyo, Fukui 910-0017 \\ **Kanebo Gohsen Ltd. Hokuriku Synthetic Fiber Plant, 47-35-1 Mizuochi, Sabae, Fukui 916-0022
}

(Received June 7, 2000)

\begin{abstract}
An optical method has been proposed and its system has been developed for measuring the change in diameter during the production of polyester filament in a manufacturing plant. The principle of the method is based on the application of scattered light on the filament. The sensor head of the system consisted of a semiconductor laser, two silicon-photodiodes as a light receiver, and a cylindrical lens. It was found from a preliminary experiment that (1) the scattered light intensity was not proportional to the diameter but to the cross section of the filament in the range between a few $\mu \mathrm{m}$ and a few tens $\mu \mathrm{m}$, (2) the measurement error for the diameter of the polyester filament was $8 \%$ for a scattered light intensity measurement error of $15 \%$, and (3) the system could be used in practical applications.
\end{abstract}

Key Words: Optical measurement, Diameter, Polyester filament, Real time

\section{Introduction}

Polyester fiber has widely been used mainly in clothes since it provides high thermal insulation, and a natural draft. In the past few years, the technique for spinning polyester fiber has been improved in terms of its quality and production efficiency. The quality of the produced filament is determined by such control factors as the temperature of polyester solution, the size of the nozzle from which a polyester solution emitted, the temperature of the cooling air in the vicinity of the nozzle, the amount of solution emitted from the nozzle, and the winding speed of the filament onto the reel. In high speed production, the winding speed is usually about $80 \mathrm{~m} / \mathrm{s}$, and the emission speed of the solution from a nozzle is about $2 \mathrm{~m} / \mathrm{s}$. In this case, a neck-like slenderizing arises in the neighborhood of the nozzle. The filament's quality and its production efficiency are determined by this slenderizing which, in turn, is determined by the above mentioned control factors. Thus, primarily as a means of process control, it is desirable to observe the slenderizing of the fiber during process in real time.

A CCD camera may be used for this purpose though it does not possess a high enough resolution for filaments as fine as a few $\mu \mathrm{m}$ in diameter. However, a scattered light intensity can successfully be used. A light scattered on a surface can collect much information regarding a cloth surface; for example, fiber density, ${ }^{1)}$ a mean fiber diameter, ${ }^{2,3)}$ and surface roughness. $\left.{ }^{4}\right)$

The paper reports an optical method for measuring the change in filament diameter during its production in a practical plant, i.e., the slenderizing in real time, and its practical system.

\section{Principle and method}

Figure 1 shows a rough schematic diagram of the spinning process. Polyester solution as the raw material of the filament is emitted from a nozzle, from which a string-like solution is formed. The string-like solution is cooled by an air current close to the nozzle and is shaped into a drawn-out filament, and is finally wounded onto a reel. In a high speed spinning process, where the emission velocity of the polyester solution is about 2 $\mathrm{m} / \mathrm{s}$ and that of the drawn-out filament is about $80 \mathrm{~m} / \mathrm{s}$, an abrupt change of the filament diameter called neck-like slenderizing arises in the neighborhood of the nozzle as shown in a cut in Fig.1. The degree of slenderizing and the position of slenderizing are determined by such control factors as the temperature and viscosity of the raw material, the emission and a winding speed, nozzle size, and cooling power. It is desirable to measure

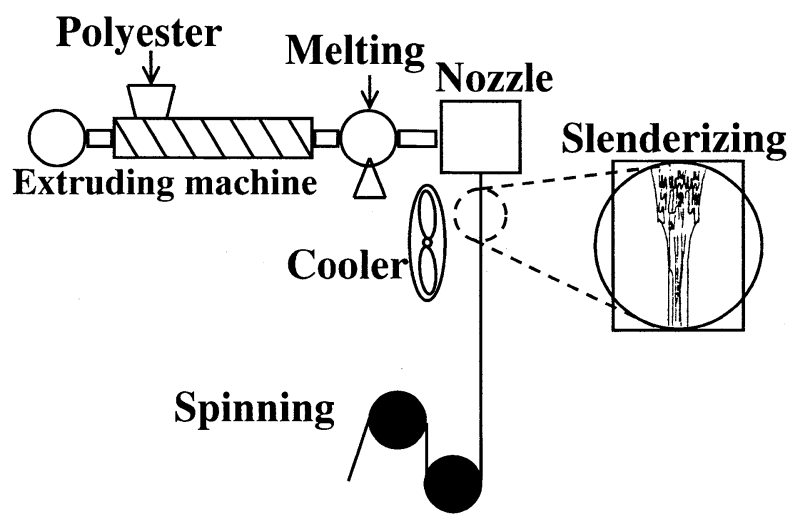

Fig.1 Schematic diagram of the spinning process. 
the change of the filament diameter at the neck-like slenderizing as a process control since the quality of the filament and its production efficiency are determined by this slenderizing.

Neck-like slenderizing does not occur with a low speed spinning process. In this case, slenderizing arises gradually over a long length of filament and such a phenomenon can not be observed. As discussed previously, ${ }^{2,3)}$ scattered light from the filament provides information regarding its surface, e.g., its diameter ${ }^{2)}$ and color. ${ }^{3)}$ To measure diameter by means of scattered light intensity, the intensity of the irradiation light and the distance from the irradiated point to the receiver should be constant. However, the fluctuation of the filament poses the most difficult problem in applying the method to a practical production process. Figure 2 shows a prototype of the optical arrangement of measuring the diameter of a polyester filament. A laser and two light receivers can be used successfully to solve both problems. In this case, the light intensity of the irradiation is constant within a laser beam. The other problem that the filament fluctuates, in other words, the change of distance between the irradiated point and the receiver, can be solved as follows: two light receivers placed on each side of the filament at an angle $\theta$ to the laser beam are used to estimate the position of the filament; one is used for front scattering light $I_{\mathrm{F}}$ and the other is used for back scattering light $I_{\mathrm{B}}$ as shown in this figure. Both scattered light intensities on the receivers, $I_{\mathrm{F}}$ and $I_{\mathrm{B}}$, are same at a chosen filament position, (2). But when a position is shifted slightly to (1) or (3) due to the fluctuation of a filament, both intensities will be different from each other as shown in a cut in Fig.2. The light intensity at a distance $R$ from a light source with an intensity $I_{\mathrm{O}}$ is given by $I=I_{\mathrm{O}} /\left(4 \pi R^{2}\right)$. An increment of the light intensity, $\Delta I$, due to an increment of the distance, $\Delta R$, can, then, be expressed as

$$
\Delta I=(-2 \Delta R / R) I .
$$

The intensity difference between both receivers at the filament positions (1) and (3), $\Delta$, is then

$$
\Delta=\Delta I_{\mathrm{F}}-\Delta I_{\mathrm{B}}=(4 \Delta R / R) I .
$$

This enables us to estimate the position of the filament. That is, in practice, we can obtain a signal from a small area around a predetermined point, i.e., (2), where both scattered intensities are almost the same within a given difference $\varepsilon, \Delta<\varepsilon$. The value of $\varepsilon$ can be determined by the measurement error of the scattered light intensity. The distance between the irradiated

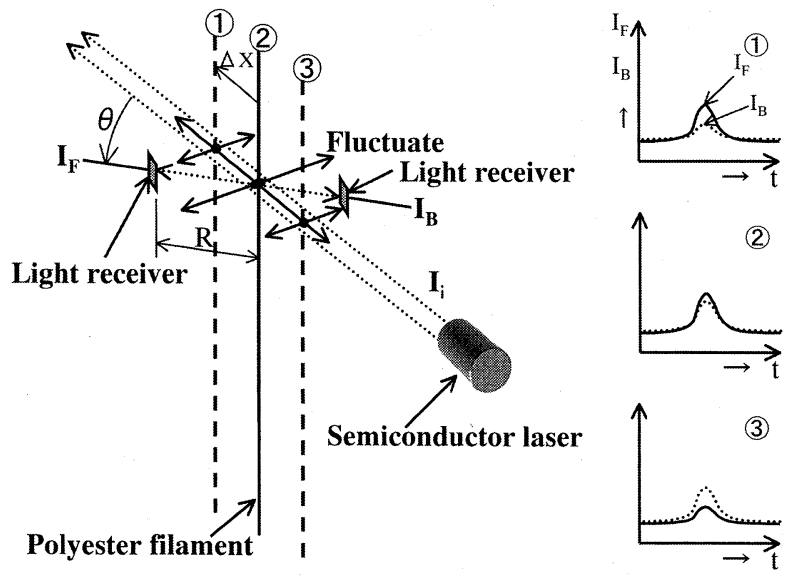

Fig.2 Prototype of the optical arrangement for measuring polyester diameter. filament and the receiver was about $R=40 \mathrm{~mm}$ from a practical point of view. The intensity change within $-1 \mathrm{~mm}<\Delta R<1 \mathrm{~mm}$ will then be $\Delta / I=0.05$ from Eq. (1). But as was shown in section 3, a dependence of scattered light intensity on a direction was larger than that of distance. We can not, therefore, determine whether or not a signal originated from $-1 \mathrm{~mm}<\Delta R<1$ $\mathrm{mm}$. This was discussed in section 3. Thus, another optical system will be required to attain higher positional sensitivity.

Figures 3 (a) and (b) show an improved optical arrangement. This configuration differs from the prototype where focused light was used; a light from a semiconductor laser was defocused by an objective lens and was then focused by a cylindrical lens. Figure 3 (a) illustrates a whole sensor head and the intensity at each position. Figure 3 (b) shows a cross section of the sheet laser. We can make the light intensity very high at the focused position, i.e., a waist position, and then detect a signal from only the waist position as follows. The laser sheet of width $2 a$ at the cylindrical lens was focused to the minimum width $2 r_{\mathrm{c}}$ at a waist position 0 . The light intensity at this position $I_{\mathrm{c}}$ is, then, increased to $I_{\mathrm{c}}=\left(r_{0} / r_{\mathrm{c}}\right) I$, where $r_{0}$ is a radius of the original laser, i.e., a width of the laser sheet, from a semiconductor laser. The light intensity at a distance $\Delta X$ from the waist position, $I(\Delta X)$, can then be expressed roughly as

$$
\begin{aligned}
& I(\Delta X)=\left(r_{\mathrm{c}} / r_{\mathrm{X}}\right) I_{c}=\left(r_{\mathrm{c}} / a\right)(L / \Delta X) I_{\mathrm{c}}=\left(r_{0} / a\right)(L / \Delta X) I, \\
& \text { where } r_{\mathrm{X}}=a \Delta X / L .
\end{aligned}
$$

In this equation, $L$ is a distance between the cylindrical lens and the waist position, and $2 r_{\mathrm{X}}$ is the width at $\Delta X$. Thus, light intensity $I$ changes inversely to the increment $\Delta X$, in contrast to the prototype arrangement shown in Fig.2. The position detection of the filament can be performed as follows. First, the light

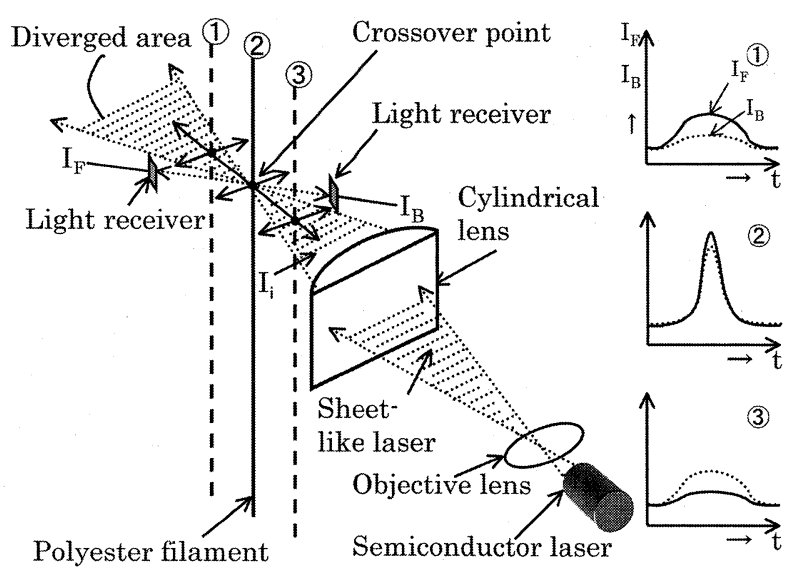

(a)

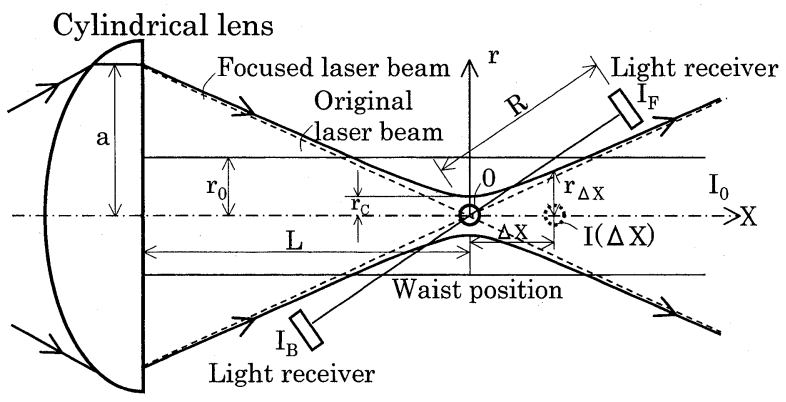

(b)

Fig.3 (a) Improved optical arrangement of the prototype, (b) cross section of the sheet laser. 
intensity scattered on a filament at the waist position, $I_{\mathrm{c}}$, was measured. Second, the intensity at any point $\Delta X, I(\Delta X)$, was measured and normalized to $I_{\mathrm{c}}, I(\Delta X) / I_{\mathrm{c}}$. Finally, only the normalized intensity greater than a given value $S$ was obtained as a signal. The value of $S$ can be determined by a tolerable maximum value of $\Delta X$ which is determined by the measurement error of the received light intensity. Thus, the improved method uses the intensity difference between $I_{\mathrm{c}}$ and $I(\Delta X)$ at a different instance of the measurement. In contrast, the previous method uses an intensity difference between $I_{\mathrm{F}}$ and $I_{\mathrm{B}}$ at the same instance. It is obvious from equation (3) that a high positional sensitivity, in other words, a large decrease in $I(\Delta X)$ due to $\Delta X$, can be obtained by small $r_{\mathrm{c}}$ and $L$ and by large $a$. The values $r_{\mathrm{c}}$ and $L$ are determined mainly by the focal length of the cylindrical lens, and small values of $r_{\mathrm{c}}$ and $L$ can be obtained by using a lens with small focal length. In our spinning process, the stringlike solution from the nozzle was, however, $100 \mu \mathrm{m}$ in diameter and that of the drawn-out filament was $15 \mu \mathrm{m}$ in diameter. The cylindrical lens with a focal length of $30 \mathrm{~mm}$ and $L \doteqdot 50 \mathrm{~mm}$ was, then, used to obtain $r_{\mathrm{c}} \doteqdot 200 \mu \mathrm{m}$ from a practical point of view. Thus, for a laser beam diameter of $1 \mathrm{~mm}\left(=2 r_{0}\right)$, i.e., laser beam width, the intensity at the waist position can be 5 times high and this leads to a high position sensitivity as discussed in 3 .

Figure 4 shows a data processing system. The received signals, $I_{\mathrm{B}}$ and $I_{\mathrm{F}}$, are amplified to an acceptable signal level for a $\mathrm{A} / \mathrm{D}$ converter and digitized by a 12-bit A/D converter. The digitized signals are processed by a personal computer and the results are displayed on the monitor. The computer has three functions: the first is to detect the signal from only a waist position by comparing $I(\Delta X)$ to $I_{\mathrm{c}}$, the second is to calculate the mean value between $I_{\mathrm{F}}$ and $I_{\mathrm{B}}$, and the last is to calculate a diameter by means of a predetermined calibration curve between the diameter and the scattered light intensity, i.e., the mean value of $I_{\mathrm{F}}$ and $I_{\mathrm{B}}$ in this case. The rate of data acquisition, i.e., the sampling frequency, was $10 \mathrm{kHz}$, which was high enough since the fluctuation frequency of the filament in the production plant was about a few $\mathrm{Hz}$.

\section{Experimental results and discussions}

Figure 5 shows an example of both scattered light intensities, $I_{\mathrm{F}}$ and $I_{\mathrm{B}}$, for the improved optical arrangement. Both intensities changed complicatedly in time. This can be explained as follows. In a practical polyester filament, a few titanic oxide particles were mixed as a brightener, which act as the light scatterer. The amount of the particles will, then, change in a mo-

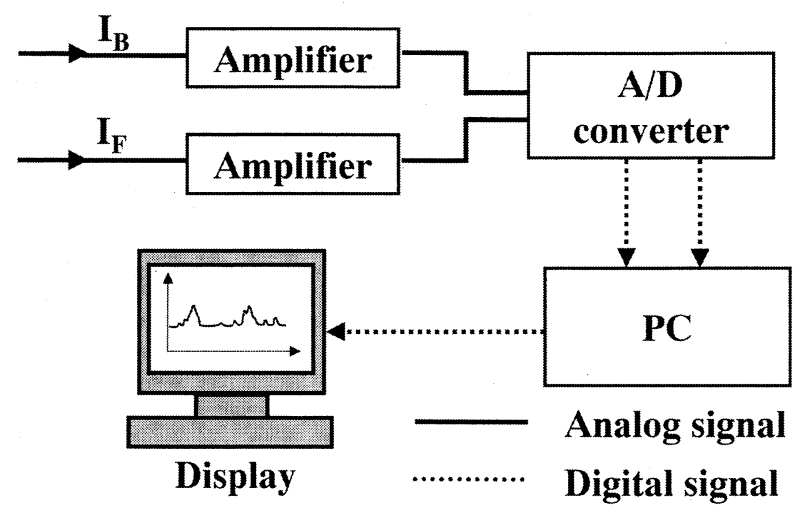

Fig.4 Data processing system.

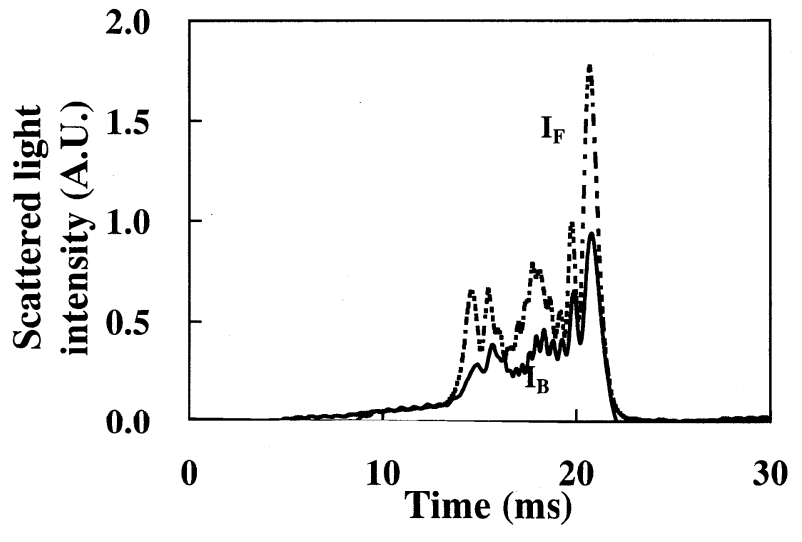

Fig.5 Scattered light intensity in real time. The date acquisition frequency was $10 \mathrm{kHz}$ and the fluctuation frequency of the filament was about $2 \mathrm{~Hz}$.

ment as the filament passes through an irradiation portion of the laser, which causes such the intensity change. These signals are, then, averaged in time and are used for the estimation of the filament diameter by the above mentioned method. The averaged light intensity was used in all the experiments in this paper.

Preliminary experiments were carried out to examine the positional sensitivity for the prototype optical arrangement shown in Fig. 2 and for the improved optical arrangement shown in Fig.3. In these experiments, all optical components were fixed and the filament position was changed to $X=-2,-1,0,1$ and $2 \mathrm{~mm}$ (Fig.3

(b)). Figure 6 shows these results. In these figures, scattered

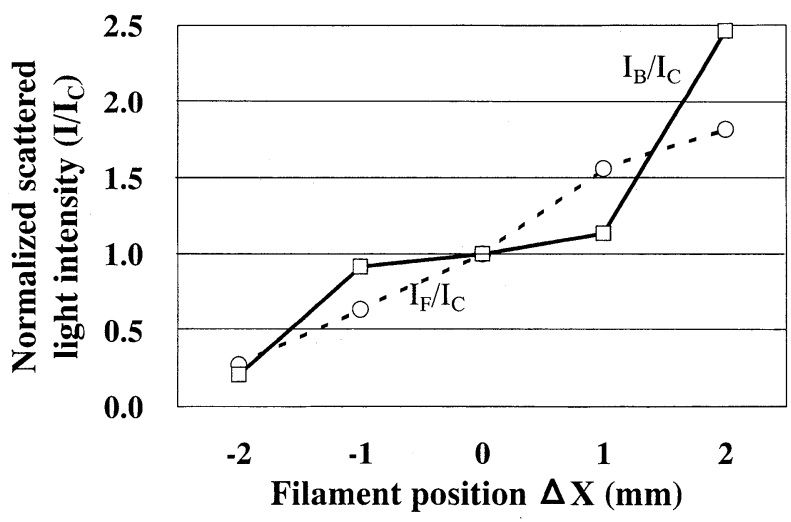

(a)

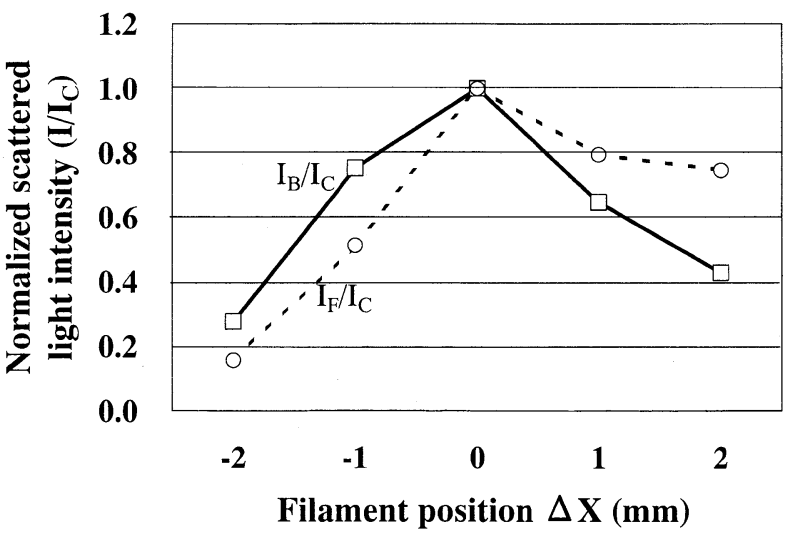

(b)

Fig.6 Scattered light intensity at various filament positions. (a) by a prototype optical arrangement, (b) by an improved optical arrangement. 
light intensities are normalized by the intensity $I_{\mathrm{c}}$ at $X=0$. Both scattered light intensities for the prototype optical arrangement in Fig. 2, $I_{\mathrm{F}}$ and $I_{\mathrm{B}}$, increased with a position shift from $-2 \mathrm{~mm}$ to $+2 \mathrm{~mm}$ (Fig.6 (a)). This will be true for $I_{\mathrm{F}}$ since the distance between the light source, i.e., a filament, and the light receiver decreases. In contrast, it seems that $I_{\mathrm{B}}$ will decrease with a position shift from $-2 \mathrm{~mm}$ to $+2 \mathrm{~mm}$ because the distance between the light source and the light receiver increases. However, the back scattered light intensity $I_{\mathrm{B}}$ increases also. This can be explained by Mie scattering. ${ }^{5)}$ In this case, the interference occurs in the scattering from a large particle and causes a directivity of the scattered light intensity, i.e., a complicated dependence of the scattered light intensity on the scattering direction. That is, the increase of the received intensity due to the increase of the directivity is larger than its decrease due to an increase of the distance. Thus, the prototype optical arrangement can not detect the correct position of the filament since both scattered intensities, $I_{\mathrm{F}}$ and $I_{\mathrm{B}}$, change in a same manner with the filament position, $\Delta X$.

Figure 6 (b) shows the normalized light intensity obtained for the improved optical arrangement. In this case, the intensity becomes maximum at a waist position, $\Delta X=0$, and decreases with both an increase and decrease of $\Delta X$, as is expected theoretically (Eq.(3)). The directivity of the scattered light intensity occurs, of course, in this case. But the intensity change due to the filament position is far larger than that due to directivity. Thus, only a signal from the waist position can be obtained by this improved optical arrangement. That is, the true signal is the one with the intensity $I_{\mathrm{s}}$ greater than $I_{\mathrm{s}}=I / I_{\mathrm{c}}-\varepsilon$, where the value of $\varepsilon$ is determined by a measurement error of the scattered light intensity. The measurement error of the scattered light intensity, $\varepsilon$, in a production plant was about $15 \%$. The maximum allowable error of the filament position $\Delta X$ was, then, about $\Delta= \pm 0.5$ $\mathrm{mm}$ from the result shown in Fig.6 (b). The positional sensitivity defined by normalized scattered light intensity $I / I_{\mathrm{c}}$ to the position change $\Delta X,\left(I / I_{\mathrm{c}}\right) / \Delta X$, was about $0.7 \mathrm{~mm}^{-1}$ as based on the

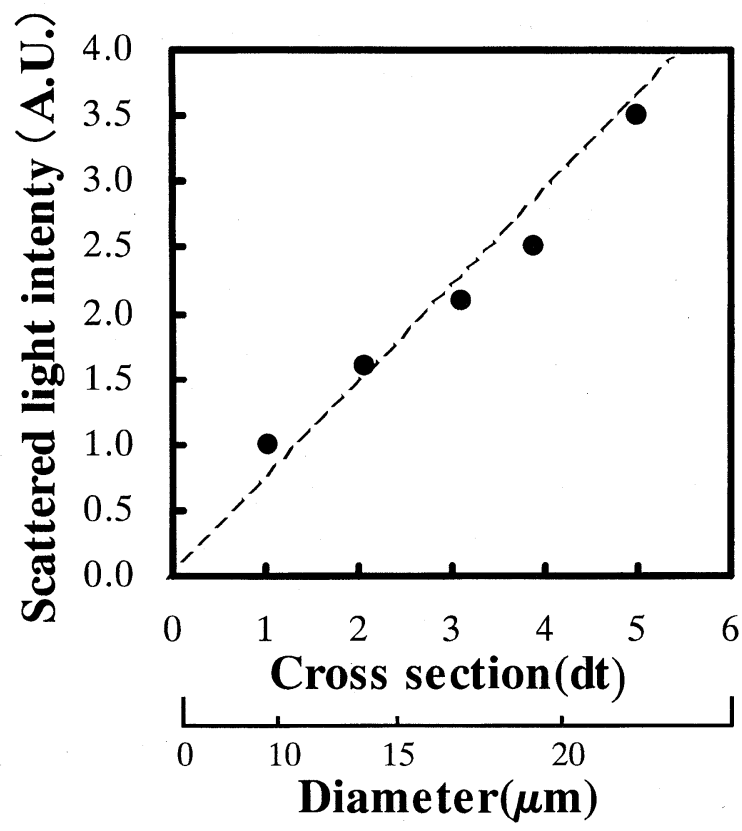

Fig.7 Relation between scattered light intensity and the cross section of the filament. An unit of dt in the axis of abscissa expresses deci-texture, where 1 texture is a unit of $1 \mathrm{~g} / 1000 \mathrm{~m}$ of the filament. results shown in Fig.6(b). That is, the signal with the intensity $I(\Delta X) / I_{c}>0.7$ will originate in the area $-1<\Delta X<1 \mathrm{~mm}$. Thus, the intensity decreases drastically with $\Delta X$ in this improved optical arrangement.

Figure 7 shows a relation between the scattered light intensity $I$ and the cross section of the filament $Q$. Thus, the light intensity $I$ was linearly proportional to the cross section of the filament, $Q$, for polyester diameter d between a few $\mu \mathrm{m}$ and a few tens of $\mu \mathrm{m}$.

$$
I=A Q=A^{\prime} d^{2} .
$$

This is rather curious because the scattered light intensity should be proportional to the irradiated surface. This is true for almost all objects where an irradiated surface acts as scattering points. However, the raw polyester that constitutes polyester filament is nearly transparent to light. In this case, a few titanic oxide particles were mixed in the polyester solution as a brightener, which act as the light scatterer. That is, the scattered light intensity is proportional to the amount of titanic oxide particles within the irradiated portion, i.e., the cross section of the filament since the thickness of the sheet-like laser light is constant. The result seen in Fig.7 was used as a calibration curve for an estimation of filament diameter. An increment of the filament diameter, $\Delta d$, due to an increment of the light intensity, $\Delta I$, can be expressed from equation (4) as,

$$
\Delta d / d=(\Delta I / I) / 2 \text {. }
$$

Thus, the measurement error for diameter is half of the measurement error for light intensity. In this experiment, the measurement error for the diameter was $\Delta d / d=0.08$ since the measurement error for the light intensity was $\Delta I / I=0.15(=\varepsilon)$.

\section{Conclusions}

An optical system has been developed for observation of the slenderizing phenomenon during the production of polyester filament. The sensor head of the system consists of a semiconductor laser and two silicon-photodiodes as light receiver. It was found from the preliminary experiment that

(1) the scattered light intensity was proportional to the cross section of the filament,

(2) the measurement error for the scattered light intensity was $15 \%$ of the maximum intensity at the waist position of the laser beam, and the measurement error for a diameter of the polyester filament was about $8 \%$,

(3) the system can be used in practical applications in production processes.

We are currently developing our system for such practical applications.

\section{References}

1) M. Ueda, M. Kawazu, J. Chen, K. Taniguchi, K. Asada, and H. Suga: Rev. Laser Eng. 26-2 (1998) 161

2) J. Chen, Y-E Lee, K. Taniguchi, K. Asada, and M. Ueda: Rev. Laser Eng. 26-2 (1998) 176.

3) J. Chen. Y-E Lee, M. Ueda, K. Taniguchi, and K. Asada: Opt. Lasers Eng. 29-1 (1998) 67.

4) J. Chen, M. Ueda, Q-P Zhang, K. Taniguchi, and K. Asada: Rev. Laser Eng. in contribution.

5) J. D. Ingle, Jr., and S. R. Crouch: Spectrochemical Analysis, Prentice Hall, Englewood Cliffs, New Jersey (1988) 498. 